\title{
Brain SPECT Imaging in Complex Psychiatric Cases: An Evidence-Based, Underutilized Tool
}

\author{
Daniel G. Amen*, ${ }^{*}$, Manuel Trujillo ${ }^{2}$, Andrew Newberg ${ }^{3}$, Kristen Willeumier ${ }^{1}$, Robert Tarzwell ${ }^{4}$, \\ Joseph C. $\mathrm{Wu}^{5}$ and Barry Chaitin ${ }^{5}$ \\ ${ }^{1}$ Amen Clinics, Inc.; ${ }^{2}$ New York University ${ }^{3}$ Thomas Jefferson University, ${ }^{4}$ University of British Columbia; ${ }^{5}$ University of \\ California, Irvine
}

\begin{abstract}
Over the past 20 years brain Single Photon Emission Computed Tomography (SPECT) imaging has developed a substantial, evidence-based foundation and is now recommended by professional societies for numerous indications relevant to psychiatric practice. Unfortunately, SPECT in clinical practice is utilized by only a handful of clinicians. This article presents a rationale for a more widespread use of SPECT in clinical practice for complex cases, and includes seven clinical applications where it may help optimize patient care.
\end{abstract}

Keywords: Brain SPECT, evidence based, brain trauma, dementia, toxicity, complex cases.

\section{INTRODUCTION}

"SPECT techniques provide a powerful window into the function of the brain and promise to become an important component of the routine clinical evaluation of patients with neurological and psychiatric diseases" [1].

This opening sentence to Holman and Devous' 1992 article "Functional Brain SPECT: The Emergence of a Powerful Clinical Method," highlighted the hope held for functional neuroimaging nearly two decades ago. A consistently growing body of research supports SPECT's clinical utility. Fourteen years ago, Vasile wrote in the Harvard Review of Psychiatry [2], "The clinical utility of SPECT in neuropsychiatry is well established." Nearly a decade ago, Camargo [3] wrote, "Brain SPECT ... is rapidly becoming a clinical tool in many places. The importance of this technique should not be overlooked, particularly in cerebrovascular diseases, dementias, epilepsy, head injury, obsessive-compulsive disorder, Gilles de la Tourette's syndrome, schizophrenia, depression, panic disorder, and drug abuse." Despite its evidence-base for multiple areas relevant to diagnosis and treatment few psychiatrists have adopted SPECT or other functional neuroimaging techniques in clinical practice.

This article lays out the evidence-based argument for the addition of SPECT for patients with complex presentations or who are treatment resistant. The article focuses on SPECT for three reasons. First, SPECT cameras are available in every major North American, European and Asian hospital making it the most widely available imaging modality available today. Second, SPECT has extensive research validating its usefulness for problems often faced by psychiatrists and is endorsed by scientific review bodies for many indications

*Address correspondence to this author at the Amen Clinics, Inc. 4019 Westerly Place Suite 100, Newport Beach, CA 92660, USA;

Tel: 949-266-3717; Fax: 949-266-3766; E-mail: docamen@amenclinic.com relevant to psychiatry. And, third, SPECT is among the least expensive neuroimaging tools and has had insurance reimbursement codes for greater than 20 years.

SPECT's prolific use in peer-reviewed research supports that it is a well-established and reliable measure of brain function (regional cerebral blood flow-rCBF) [4]. Both the American College of Radiology [5] and the European Society of Nuclear Medicine (ESNM) [6] have published similar evidence-based guidelines for using SPECT to enhance patient care. Commonly accepted clinical indications for SPECT include:

- Evaluating patients for cerebrovascular disease.

- Evaluating patients with suspected dementia including early detection, differential diagnosis, and in the predementia phase.

- Presurgical localization of epileptic foci.

- Evaluation of traumatic brain injury, especially in the absence of computed tomography (CT) and/or magnetic resonance imaging (MRI) findings.

- Evaluation of suspected inflammation to provide helpful information in progressive inflammatory disorders including viral encephalitis, vasculitis, and HIVencephalopathy.

- Assessing brain death.

It is obvious to practicing psychiatrists that all of these indications, except perhaps brain death and the pre-surgical location of epileptic foci, are potentially valuable in clinical practice. Psychiatrists commonly evaluate and treat dementia and the consequences of brain trauma, cerebral vascular disease, inflammation and infections. Besides these common indications, the ESNM guidelines also state, "SPECT can be useful in other indications such as movement disorders and psychiatric diseases (e.g. for follow-up of depression)." 


\section{SPECT BASICS}

SPECT uses radioisotopes bound to neurospecific pharmaceuticals to evaluate $\mathrm{rCBF}$ and indirectly metabolic activity. Hexamethylpropyleneamine oximine (HMPAO) and ethylcysteinate dimer (ECD) are two commonly available FDA-approved radiopharmaceuticals; both provide regional cerebral blood flow (rCBF) images where the patient acts as his own control. A normal SPECT scan shows full, even, symmetrical perfusion [7], with the most intense perfusion in the cerebellum with HMPAO [8] and in the occipital lobes with ECD [9]. In reading SPECT images, experienced clinicians look for symmetry and areas of increased and decreased perfusion. See Figs. (1) and (2) for examples of healthy HMPAO scans. When evaluating SPECT, it is important to know the age of the patient. rCBF changes significantly over time. Children tend to have very active brains, older people less so [10].

\section{SPECT HAS A SERIOUS IMAGE PROBLEM}

SPECT has a serious 'image' problem related to many clinicians' misunderstanding of its advantages and disadvantages. Five areas contribute to this problem:

1. There is a widely held idea that the clinical use of SPECT is restricted by limited resolution. Early SPECT practice utilized single-headed cameras that produced lower resolution images of about $12 \mathrm{~mm}$. However, sophisticated multi-headed gamma detectors with fan beam collimators have been available for the past two decades and can produce images with a resolution of $6 \mathrm{~mm}$. George reported that multi-head SPECT camera resolution is similar to PET at considerably less cost [11]. Multi-headed SPECT cameras cost between $\$ 100,000$ for refurbished systems to $\$ 400,000$ for new systems while PET/CT cameras cost between $\$ 1.5$ to 3 million dollars [12]. This cost is then reflected in the price of the scans, with a SPECT scan averaging $\$ 1,100$ versus a PET scan starting at $\$ 3,800$.

2. Many nuclear medicine departments provide minuscule gray scale horizontal, coronal, and sagittal SPECT slices. The poor quality images are hard to read and understand. When clinicians cannot easily understand the images, they dismiss the technology as useless. Several current manufacturers provide software for three-dimensional image renderings that allow for more easily understandable images, such as those used in this article. Even though it is always necessary to evaluate the sliced images for appropriate detail, clinicians and patients need images that they can understand.

3. Functional neuroimaging training seldom combines radiologists and psychiatrists. Since psychiatry has yet to embrace this technology, many radiologists and nuclear medicine physicians have little to no training or experience in utilizing SPECT for neuropsychiatric indications. Even though the SPECT literature is replete with studies showing areas of increased perfusion in a number of psychiatric diagnoses and epilepsy most radiologists or nuclear physicians report only areas of decreased perfusion. More education and collaboration is needed among specialties.
4. Another common SPECT criticism is radiation exposure, especially in children. However, the average radiation exposure for one SPECT scan is 0.7 rem (i.e., similar to a nuclear bone scan or head CT) and is considered safe. The Health Physics Society states, "There is no direct evidence of radiation ever causing any harm at the exposure levels encountered with diagnostic radiological examinations "[13]. The benefit-risk ratio of such radiation exposure needs to be evaluated against the considerable costs (monetary, and in terms of suffering and dysfunction) imposed by treatment resistant psychiatric disorders.

5. A final concern is why use SPECT over other brain imaging procedures that do not require ionizing radiation, namely perfusion/arterial spin labeling MRI and quantitative electroencephalography (QEEG). Perfusion/arterial spin labeling MRI is advantageous in that it doesn't use ionizing radiation (it uses magnetically labeled endogenous blood water) and is minimally invasive; however, it is limited based on its low-signal-to-noise ratio and low temporal resolution. An alternative brain imaging modality, QEEG, does not require radiation and is low in cost (\$600), however, it is limited to measuring brain wave activity at the scalp, and gives limited information regarding subcortical structures.

\section{CLINICAL APPLICATIONS OF BRAIN SPECT IMAGING}

Several important patterns have emerged from SPECT research that has direct application to day-to-day clinical practice. Here are 7 examples:

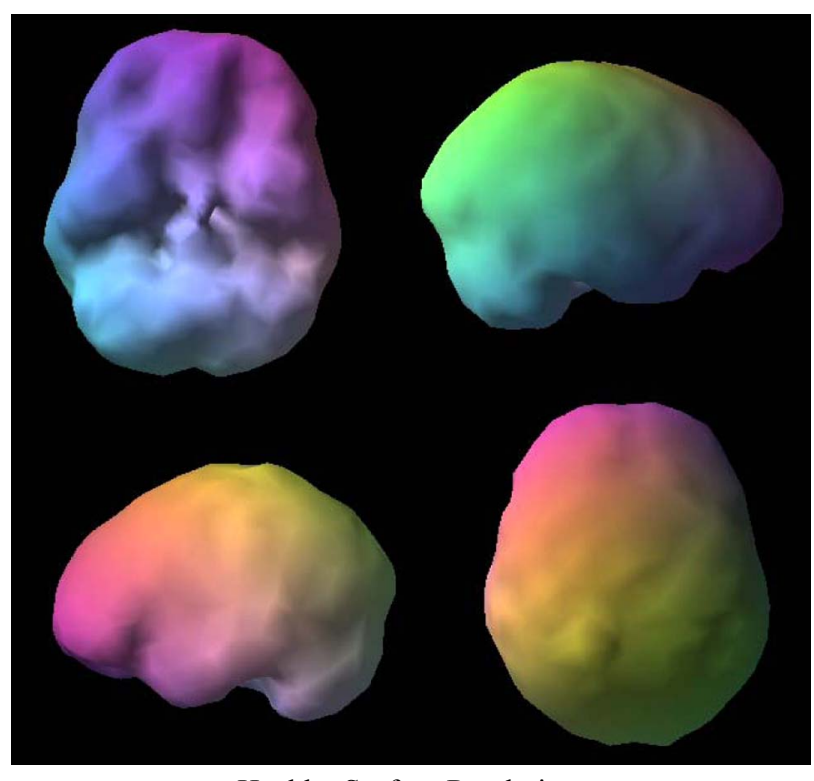

Healthy Surface Rendering.

Fig. (1). Shows a healthy 3D surface rendering of SPECT information, looking at the top $45 \%$ of brain perfusion, anything below that level shows up as a hole or a dent. The holes do not mean no perfusion, they mean low perfusion, compared to a healthy dataset. Activity below the top forty-fifth percentile is 3 standard deviations below normal [10]. A healthy scan shows full, even, symmetrical perfusion. 


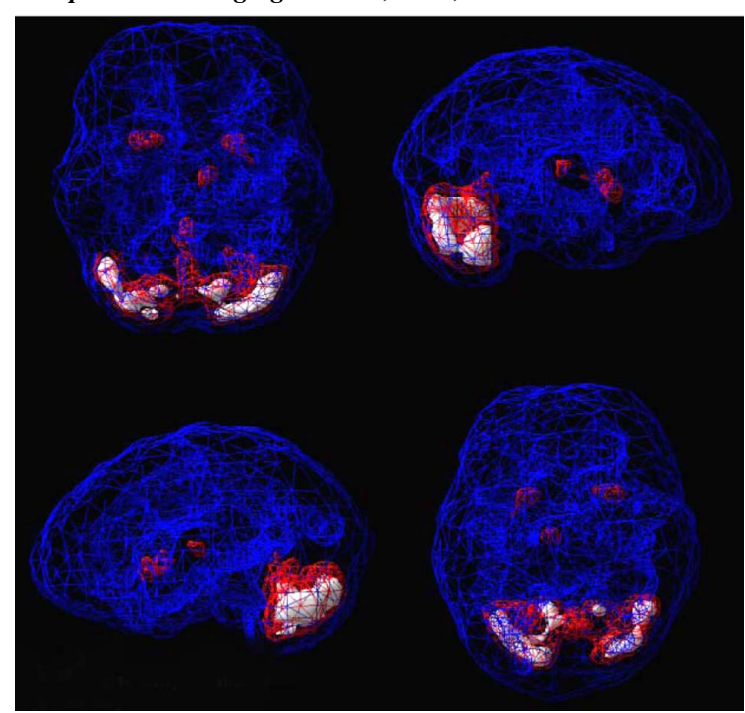

Healthy Active Rendering.

Fig. (2) Shows a healthy 3D active rendering of SPECT information, looking at areas of increased perfusion. Blue equals average perfusion, red equals the top $15 \%$ of perfusion and white is the top $8 \%$. Activity above these levels is 2 and 3 standard deviations above normal outside the cerebellum(10). A healthy active scan with HMPAO shows increased perfusion in the cerebellum.

\section{Overall Decreased Perfusion}

This pattern is often associated with toxicity, illness or insult to the brain. It is frequently seen in drug and alcohol abuse, abuse of prescription medications such as benzodiazepines, environmental toxins, such as carbon monoxide poisoning, infectious disease, such as meningitis, anoxic states, significant hypothyroidism, anemia, chemotherapy and severe dehydration. Seeing this pattern does not give the etiology, but alerts clinicians to search for causes which will allow them to understand it.

Here is an example: A couple came for evaluation after their marital therapist told them she thought they should get divorced. They'd been treated for three years and spent nearly $\$ 20,000$ in care. The husband's diagnosis was mixed personality disorder with narcissistic and antisocial features. The couple's family physician recommended another opinion that included SPECT scans. The husband's scan showed overall decreased perfusion (Fig. 3). The husband reported that he didn't drink alcohol and had never used drugs, which his wife confirmed. The scan results caused his physician to think through a completely different differential diagnosis beyond personality disorder. It turned out that the husband worked in a furniture factory, finishing cabinets. The inhalants found in finishing products may compromise brain functioning and show a toxic pattern on scans [14]. Marital therapy is destined to fail until the husband is removed from the toxic environment. This information significantly changed the treatment plan and was instrumental in helping the couple's marriage.

\section{Traumatic Brain Injury (TBI) Patterns}

TBI is a major public health concern. TBI survivors commonly face a range of psychiatric disorders, affecting functional status, cognition, and mood [15]. Of course, not everyone with a significant brain injury has lasting symptoms. Those who do, however, present daunting problems of differential diagnosis. But how does a psychiatrist know about individual patients and brain injury unless they look? Relying on clinical history alone is inadequate. Even upon extensive questioning, many patients forget that they have sustained a significant brain injury. The following case demonstrates this frustrating problem:

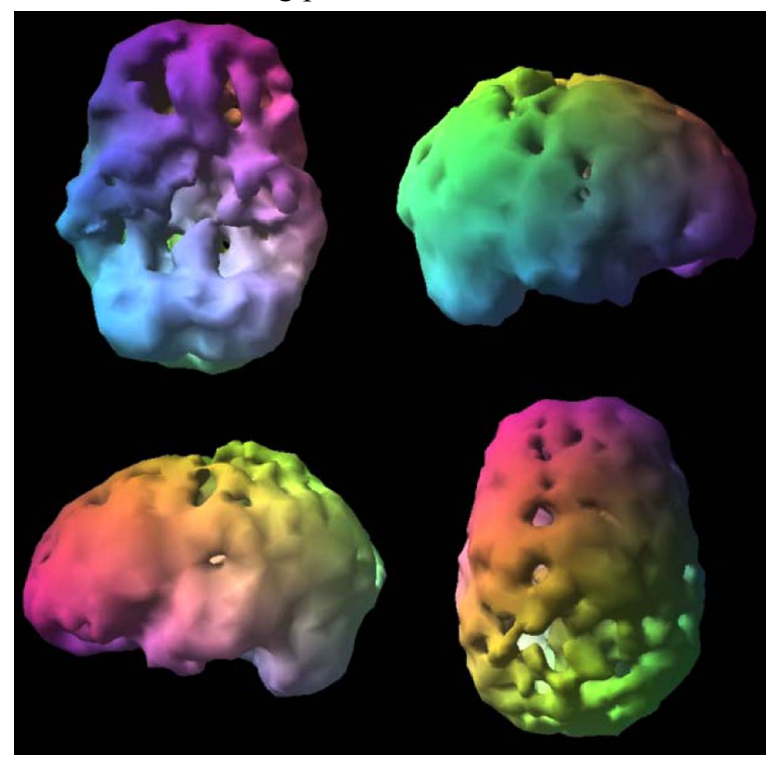

Fig. (3). Toxic Surface Scan notice the "Swiss cheese, shriveled appearance, indicating areas of decreased perfusion.

A 26 year old male patient with severe impulsivity and depression in a drug treatment program was asked 10 times, with extensive examples, whether or not he had a brain injury. He said no each time. His SPECT scan (Fig. 4), however, showed evidence of substantial hypoperfusion, consistent with trauma in the left frontal-temporal lobe region. When asked again, he then remembered a motorcycle accident where he broke his left jaw, near the site of his SPECT deficit.

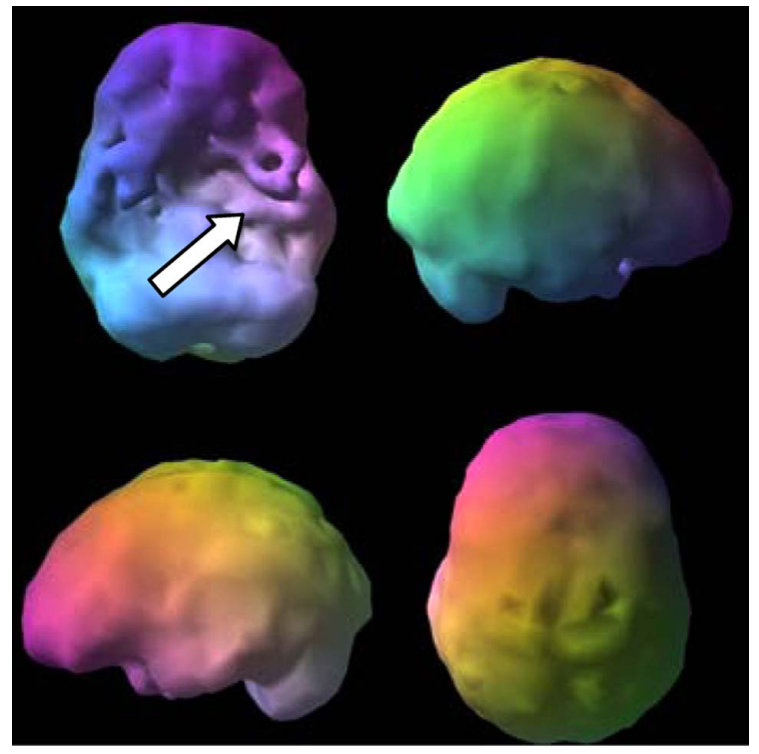

Fig. (4). Trauma. Asymmetrical decreased perfusion left frontal temporal lobe. 
SPECT can help identify if trauma is present and which brain system or systems are affected. Common findings in trauma on SPECT include:

- focal decreased near sight of injury and/or opposite side (contra coup)

- asymmetrical hypoperfusion in the prefrontal, temporal, parietal or occipital lobes

- flattening of the prefrontal pole

- decreased anterior temporal poles

- decreased contralateral cerebellar perfusion.

SPECT aids in understanding TBI patients' symptomatology and assists clinicians in developing targeted treatment strategies [16, 17]. For example, decreased prefrontal cortex perfusion is often associated with executive dysfunction and may be helped with psychostimulants or other strategies to enhance frontal lobe function; whereas decreased temporal lobe perfusion is often associated with irritability and mood instability and may be helped with anticonvulsant medication. Literature indicates that SPECT helps evaluate perfusion abnormalities not only in blunt brain trauma, but also in cases of post-concussive syndrome and whiplash [18]. Brain injured patients with normal EEG, CT, and/or MRI scans often complain of headaches, memory loss, concentration difficulties, dizziness, perceptual sensitivities, and emotional lability. Such patients may be labeled as malingering, when there are significant and demonstrable functional abnormalities present. Researchers investigating the differences between functional and structural imaging techniques have found SPECT to be more sensitive for patients with varying degrees of head trauma [19].

Researchers have also compared the differences between functional and structural imaging in relation to clinical outcome and prognosis. Jacobs et al. [16] used SPECT to prospectively evaluate 67 mild-to-moderate brain injured patients. Each patient had a clinical evaluation and SPECT scan within four weeks of the initial injury and three months after the first scan. Of the 33 patients who showed no significant abnormalities on their initial scan, 97\% resolved their clinical symptoms within three months. By contrast, in the 34 patients with abnormal initial SPECT scans, 59\% continued to experience significant clinical symptoms three months later. The positive predictive value of an abnormal initial scan was only 20/34 (59\%), but if the second scan 12 months later was also abnormal the sensitivity for the repeat SPECT was 19/20 (95\%). These authors suggest that negative initial SPECT studies are a reliable predictor of a favorable clinical outcome.

SPECT assists in the diagnosis, prognosis, and treatment of TBI patients. SPECT may also help uncover brain trauma in clinically confusing or complex cases because patients often fail to report or forget about significant brain injuries, perhaps due to peri-traumatic amnesia. A limitation for SPECT's use in brain trauma is that typically no prior SPECT study is available for comparison. Therefore, it is often not possible to date the trauma with neuroimaging. Remote trauma from childhood often presents neuroimaging findings similar to those seen in more recent trauma.

\section{Evaluating Cognitive Decline}

To date, autopsy reports have been the "gold standard for an Alzheimer's Disease (AD) diagnosis." However, research suggests that SPECT, when used in conjunction with clinical history and other diagnostic tests, is helpful when evaluating patients who are experiencing cognitive decline [20] and should be used to help differentiate $A D$, vascular dementia (VaD), frontal lobe dementia (FLD), suspected Lewy Body Dementia (LBD), normal pressure hydrocephalus (NPH) and pseudodementia (PSD) if the diagnosis is in doubt [21]. The functional brain imaging patterns associated with AD include decreases in the posterior cingulate gyrus, parietal and medial temporal lobes; FLD include frontal and temporal lobe deficits; $\mathrm{VaD}$, a vascular pattern of decreased activity in multiple areas; LBD often shows decreased occipital lobe perfusion; NPH is associated with enlarged ventricles out of proportion to cortical atrophy; and PSD often shows an absence of dementia patterns with increased limbic activity [22]. This differential is critical because the use of high potency antipsychotics in patients with LBD, which often affects the occipital lobes on SPECT [23], can cause severe and sometimes irreversible deterioration. Patients diagnosed with different dementia disorders often present with similar symptomatology (e.g., social withdrawal and behavioral disinhibition). Because treatments for these disorders vary, differential diagnosis is critical, particularly since some dementia patients will have a reversible cause, such as depression or normal pressure hydrocephalus, or a cause they may be significantly stabilized with aggressive intervention, such as VaD.

Here is a case example: L, a 73 year old male, suffered with persistent memory problems. His neurologist, without the benefit of imaging, diagnosed him with AD. Memantine and donezepil had no effect. Two years later, his family brought him for further evaluation. His SPECT scan showed significantly enlarged ventricles, without concomitant cortical atrophy (Fig. 5). His scan did not show the typical low posterior cingulate and bilateral temporal-parietal hypoperfusion, consistent with AD. Given the pattern, and subsequent MRI, he was diagnosed with normal pressure hydrocephalus (NPH). After a shunt his memory markedly improved.

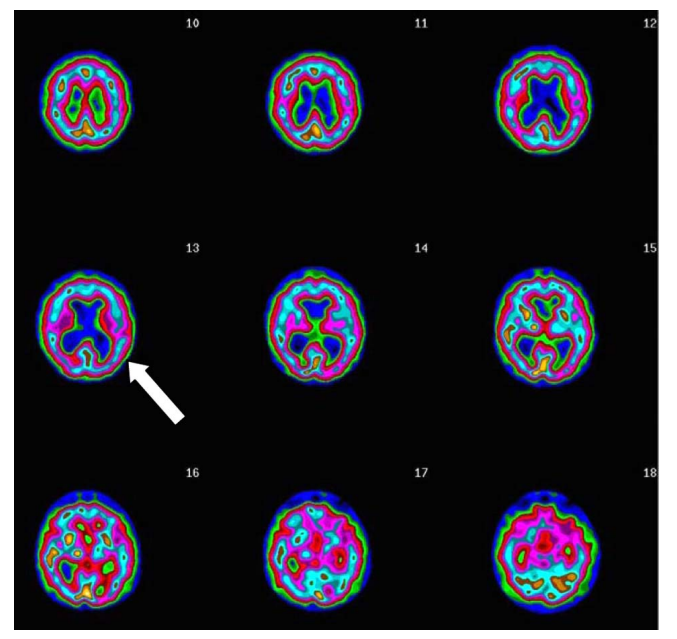

Fig. (5). Transaxial Slices. Slices 12 and 13 show inverted lobster pattern associated with ventricular enlargement. 
Some would argue that an MRI would be a more appropriate study in evaluating NPH, which the authors agree. Yet, functional imaging studies like SPECT provide a wealth of other data, such as giving information on areas of increased and decreased perfusion, in addition to enlarged ventricles, making it is useful screening tool. For example, Bonte et al. [24] performed SPECT studies in patients with possible dementia and compared them to images of an elderly healthy control group. Histopathologic correlation was available in 54 patients (with autopsy in 51, with biopsy in three). The results of the study showed SPECT diagnoses were true-positive in 37, true-negative in eight, false-positive in three, and false-negative in six patients. Sensitivity was $86 \%$; specificity, $73 \%$ and the positive predictive value was $92 \%$, with an accuracy of $83 \%$. The authors concluded that SPECT assists in the early and late diagnoses of AD and in the differential-diagnosis of the dementias when there is a complicated or confusing clinical picture.

\section{Negative Emotionality and Hyperfrontality}

Hyperfrontality, or increased perfusion in the prefrontal cortex and anterior cingulate gyrus, is associated with a number of different psychiatric illnesses that have a common theme of cognitive inflexibility or getting stuck on negative thoughts or negative behaviors, such as in obsessive compulsive disorder (OCD) [25, 26], autism, posttraumatic stress disorder, and certain types of anxiety and mood disorders $[27,28]$. This pattern is often seen in patients who struggle with being rigid, inflexible and oppositional [29], and crosses several different diagnostic groups.

When SPECT scans detect the hyperfrontality pattern, it opens new avenues for intervention since this finding has been associated with predicting a positive treatment response to serotonergic medication in depression [30-34] and OCD $[35,36]$, predicting a positive response to sleep deprivation $[37,38]$ and repetitive transcranial magnetic stimulation[39] for depression, predicting treatment response to a cingulotomy in OCD [40], and help in distinguishing OCD from ADHD [41].

Hyperfrontality will never give a classic DSM diagnosis per se, but rather it provides new directions for treatment by providing a picture of the brain physiology underlying the clinical presentation.

Here is a clinical example. V, a 17 year old male, had severe temporal lobe epilepsy. He also had aggression that was non-responsive to behavioral interventions. His SPECT scan showed severely decreased perfusion in his left temporal lobe (Fig. 6) consistent with epilepsy, and he showed severely increased perfusion in the anterior cingulate gyrus and lateral frontal lobes (Fig. 7), a pattern consistent with OCD. Even though $\mathrm{V}$ did not have clear OCD symptoms, he was rigid, inflexible and easily upset when something did not go his way. With the addition of sertraline, an antidepressant that tends to lower $\mathrm{rCBF}$ in this brain region [31], his behavior significantly improved. Managing $\mathrm{V}$ without the benefit of scans handicapped the doctor, patient and family.

\section{Behavioral Problems and Hypofrontality}

Hypofrontality, decreased perfusion or activity in the prefrontal cortex, is another important SPECT finding that is often helpful in understanding and targeting treatment in individual patients. Hypofrontality is associated with a negative response to serotonergic medication in depression [30] and clozapine in schizophrenia [42] as well as with predicting relapse in alcoholics [43], improved response to acetylcholine-esterase inhibitors for memory and behavior in AD $[44,45]$, predicting a poor response to ketamine in fibromyalgia patients [46] and improved response to stimulants in patients with ADHD symptoms during a concentration challenge [47]. Hypofrontality is also associated with antisocial symptoms, impulsive behaviors, and murder [48, 49] as well as with completed suicide, which is often an impulsive act [50]. When hypofrontality is present in depressed patients, it is important to be vigilant in their care, as well as involve family support, as they may be less likely to respond to typical antidepressant medications and they may not have the cognitive resources to follow through with recommendations.

Given its broad range of involvement in different types of psychiatric illness hypofrontality, like other findings, will not give a specific psychiatric diagnosis, but it allows the clinician to know the underlying pathophysiology of the patient's presenting problem and can help explain issues with cognitive impairment or behavioral problems.

\section{Mood Instability, Memory Problems and Temporal Lobe Abnormalities}

Evaluating temporal lobe function is important in psychiatric medicine, especially as it relates to the evaluation of patients with memory problems, mood instability, aggression, and receptive and expressive language [51]. As described above, the temporal lobes are commonly affected in brain injury. Their function however cannot be evaluated simply by clinical phenomenology. SPECT offers a reliable tool to evaluate temporal lobe function. According to Devous et al. "Both SPECT and PET have localizing power

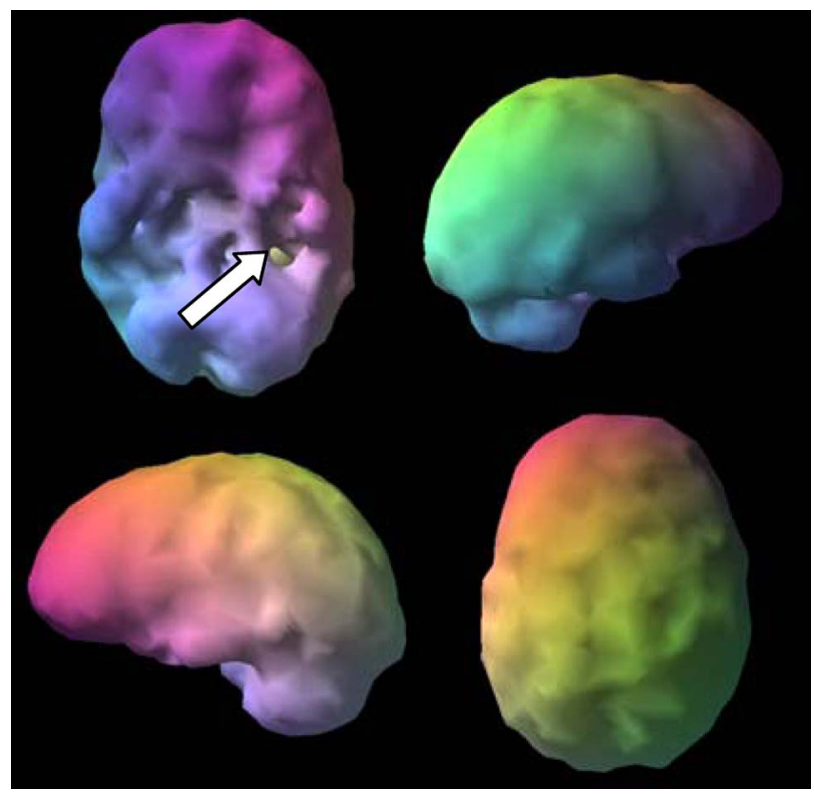

Fig. (6). Outside Surface Scan severe left temporal lobe hypoperfusion (arrow). 


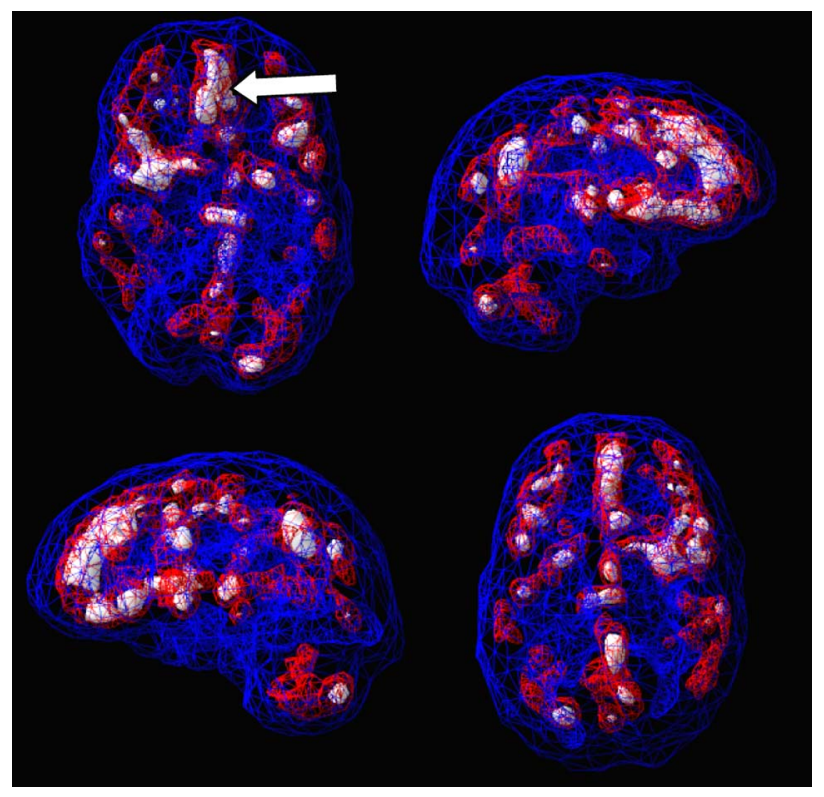

Fig. (7). Active scan severe hyperfrontality (arrow).

approaching that of combined scalp and depth EEG."[52] Patients with epilepsy have high psychiatric comorbidity [53], and psychiatrists increasingly rely on the use of anticonvulsants as 'mood stabilizers,' which are shown to stabilize or calm overall brain activity and perfusion, especially in the temporal lobes $[54,55]$.

Temporal lobe epilepsy (TLE) is one of the most frequently encountered chronic epileptic disorders and is associated with numerous psychiatric symptoms, such as depressed mood, anergia, irritability, euphoric mood, atypical pain, insomnia, fear, and anxiety [56]. The medial temporal lobes are frequently involved in TLE and they are difficult to evaluate with routine EEG studies and therefore may be missed. SPECT findings in epilepsy most often reveal focal decreased perfusion in the interictal phase and focal increased perfusion in the seizure's ictal phase. SPECT adds to the clinical evaluation of temporal lobe function by identifying area(s) of abnormality and showing deficits not seen by EEG.

Based on the authors' clinical experience, when abnormalities in the temporal lobes are seen (either low or high perfusion) and mood instability or temper problems are present, anticonvulsants provide a rational treatment option [57]. If there are memory or learning issues (and low temporal lobe perfusion), acetylcholine-esterase inhibitors may be helpful [58], always taking into consideration the clinical picture.

Here is an example: C, age 12, was hospitalized for attacking another child at school. This was his third psychiatric hospitalization. C had been diagnosed with ADHD at age 6, but stimulants caused him to hallucinate. At age 9 he was hospitalized for an aggressive episode, diagnosed with a mood disorder and placed on an antidepressant without benefit. By age 12 he had been in psychotherapy for 3 years, also without benefit. Given his resistant illness, and the tens of thousands of dollars spent on his care, a brain SPECT study was ordered which showed severe left temporal lobe hypoperfusion (Fig. 8). Unilateral temporal lobe hypoperfusion is often seen with epileptic phenomena [52]. On an anticonvulsant C's behavior normalized and he has maintained his progress for over 10 years.

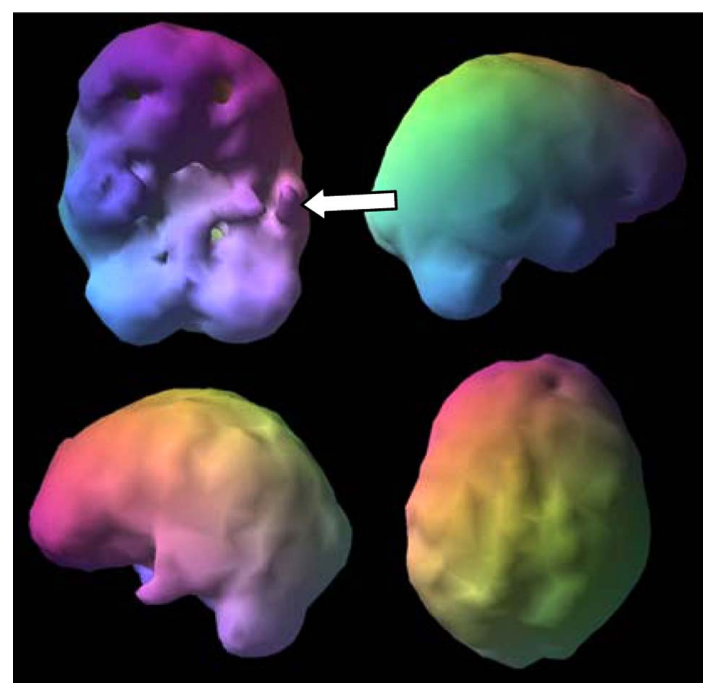

Fig. (8). C's Brain SPECT Study severe hypoperfusion left temporal lobe (arrow)

\section{Subtyping Dimensional Behavior to Predict Treatment Response}

SPECT's use in clinical practice dovetails closely with the spirit of NIMH's RDoC initiative which looks to develop new ways of classifying psychopathology based on observable behavior and neurobiological measures [59]. In order to construct such a system, it is necessary to obtain accurate measures of the functioning of the brain systems which support various normal and abnormal emotions, undergird critical functions such as attention and memory, and modulate functions such as aggression. SPECT scans have the possibility of facilitating the subtyping of mental illnesses, such as depression, ADHD, obsessive-compulsive disorder, and aggression based on brain system pathophysiology.

\section{SUBTYPING AGGRESSION}

The role of brain function in aggression is one of NIMH's five major domains of interest and an area where SPECT imaging may provide immediate clinical help. From the authors' work with 75 murderers, and hundreds of patients who have exhibited serious aggressive behavior ranging from assault, robbery, rape, kidnapping, arson, bombings, to road rage, and stalking, we have seen that aggressive behavior is not defined by one single brain finding, but rather clusters in at least three different patterns. The first is impulsive aggression, associated with hypofrontality, a finding reported in antisocial personality disorder $[60,61]$. $\mathrm{Pa}-$ tients with a compromised prefrontal cortex are frequently unable to regulate aggressive impulses and are at higher risk for exhibiting violent activity than other patients. The second cluster we have labeled 'compulsive aggression,' often associated with hyperfrontality, where people act out because they cannot get negative thoughts out of their minds, or because they are extremely rigid and inflexible. The third clus- 
ter of aggressive patients we've seen have SPECT findings of temporal lobe abnormalities. Soderstrom et al. [62] retrospectively evaluated violent subjects' SPECT and MRI scans and compared them to the SPECT results obtained from a group of volunteers who had no history of a neurological or psychiatric disorder, head trauma, or metabolic disease. Sixteen of the 21 impulsively violent subjects showed some hypoperfusion in the temporal and/or frontal lobes upon visual inspection. By contrast, MRI failed to show any corresponding structural damage or abnormalities in this sample of violent offenders. Another study of recidivistic violent offenders [63] revealed that the largest functional deficits in their violent patients were found in the temporal lobe. These abnormalities were similarly found in subjects whose structural CT scans were normal. Knowing a violent patient's underlying brain pattern may be helpful to getting them the right help. In the authors' experience we have often seen multiple patterns associated with violence, such as low prefrontal cortex and temporal lobe perfusion.

Here are two examples of 15 year old males, multiple murderers, who had significantly different SPECT patterns, which would lead to different clinical management decisions.

KK was diagnosed with ADHD, dyslexia and depression. He had been evaluated by two psychiatrists and placed on a psychostimulant and SSRI without benefit. He was arrested after bringing weapons to his high school. After he was released from jail he murdered his mother and father and then went back to his high school the next morning and shot 24 people, killing two more. As part of his pre-trial evaluation, a SPECT scan was performed and showed severe overall decreased perfusion, especially in the area of the left medial temporal lobe and inferior orbital prefrontal cortex. The scan was consistent with prior toxic exposure, anoxia or infection. The SPECT scan and history raise the possibility that an anticonvulsant (given the severe decreased left temporal lobe perfusion) followed by a psychostimulant (to enhance his low prefrontal cortex perfusion) was worth considering to help modulate his aggressive impulses.

PP had been diagnosed with ADHD and dyslexia. In a rage he murdered his mother and eight year old sister. PP's SPECT scan showed significant hyperfrontality, especially in the anterior cingulate gyrus. As noted above, this presentation is more likely to respond to an SSRI.

Here is the same symptom presentation, multiple murder, but very different brain patterns. The differences in the scan patterns give suggestions for different treatment regimens.

\section{SUBTYPING DEPRESSION}

Anxiety and depressive disorders are two of the most common psychiatric problems worldwide and subtyping these disorders using brain system pathology is an important step in improving treatment outcomes [64]. Functional imaging, including SPECT, helps to elucidate this process in individual patients. As noted above, hyperfrontality is associated with a positive response to SSRIs in depressed and anxious patients, while hypofrontality is associated with a negative response, and has been reported in completed suicides. Little et al., on the other hand, found that lower prefrontal activity prior to treatment was associated with a positive response to buproprion and venlafaxine [65]. These medications involve enhancing dopamine and norepinephrine, which in the authors'experience, tend to enhance prefrontal cortex activity, while SSRIs tend to be calming [31]. Up to 50 or $60 \%$ of patients with chronic epilepsy have various mood disorders including depression and anxiety [66, 67]. Using SPECT gives the clinician a more rational reason to use anticonvulsants when focal areas of increased or decreased perfusion are seen, especially in the temporal regions. Likewise, if overall decreased perfusion is present, it alerts the clinician to look for toxic or metabolic causes of the problem, or if a brain trauma pattern is seen, commonly associated with anxiety and mood disorders, this can be investigated further as well.

\section{CONCLUSION}

There are a number of important areas where SPECT has the potential to provide relevant information to help personalize treatment to patients' specific brain system pathophysiology rather than rely solely on general diagnostic and/or therapeutic categories. In this paper we described 7 such areas. Furthermore, in the authors' experience, another immediate benefit of using brain SPECT imaging is that the patient's and family's guilt, shame and stigma are significantly reduced as they see their illness as medical rather than moral with concurrent improvements in treatment compliance. In the authors' opinion, brain SPECT or any other neuroimaging modality should always occur in conjunction with clinical assessment since it is not the isolated diagnostic accuracy that has greatest importance but rather the value added to routine clinical assessment.

Over the last quarter century, there has been much discussion of the "Future of Psychiatry" [68] as we move into a more biologically based paradigm. How would we maintain our subjective patient focus within this new framework? Reynolds, Lewis, Detre, Schatzberg and Kupfer [69] have weighed in on this work in progress. One thing that is becoming very clear is that our resident trainees may require more exposure to translational neuroscience during their early years of residency and neuroimaging needs to be an important component of this curriculum. A recent report demonstrated the integration of a neuroimaging module within a residency program had wide approval by residents. Other programs have experimented with variations but within the neurology rotation of the PGY-1 year. The Psychiatry RRC of the ACGME will need to take this on in earnest for the development of the field.

This is an exciting time in the history of psychiatry, as the field will likely significantly change over the next 30 years to incorporate functional neuroimaging in clinical practice. Functional magnetic resonance imaging (fMRI) among other techniques are often seen as proving the future of neuroimaging. Yet these techniques are much more expensive and are more technically difficult to administer. The authors' hope is that SPECT does not get left out because of a lack of understanding or training in the use of this valuable tool.

\section{REFERENCES}

[1] Holman BL, Devous MD, Sr. Functional brain SPECT: the emergence of a powerful clinical method. J Nucl Med 1992; 33: 1888-904. 
[2] Vasile RG. Single photon emission computed tomography in psychiatry: current perspectives. Harv Rev Psychiatry 1996; 4: 2738 .

[3] Camargo EE. Brain SPECT in neurology and psychiatry. J Nucl Med. 2001; 42: 611-23.

[4] Amen Clinics, Inc. SPECT research articles and abstracts. Available from: http://www.amenclinics.com/brain-science/spectresearch/spect-abstracts.

[5] Graham M. Guidelines and Standards Committee, Comments Reconciliation Committee. American College of Radiology Practice Guideline for the Performance of Single Photon Emission Computed Tomography (SPECT) Brain Perfusion and Brain Death Studies 2007; 1-6. Available from: http://www.acr.org/SecondaryMainMenuCategories/quality_safety/guidelines/nuc_med/ct_spect_ brain_perfusion.aspx.

[6] Kapucu OL, Nobili F, Varrone A, et al. EANM procedure guideline for brain perfusion SPECT using (99m)Tc-labelled radiopharmaceuticals, Version 2. Eur J Nucl Med Mol Imaging 2009; 36: 2093-102.

[7] Datz FL, Patch GG, Arias JM, Morton KA. Nuclear Medicine: A teaching profile. St.Louis, MO: Mosby Yearbook 1992.

[8] Catafau AM, Lomena FJ, Pavia J, et al. Regional cerebral blood flow pattern in normal young and aged volunteers: a 99mTcHMPAO SPET study. Eur J Nucl Med 1996; 23: 1329-37.

[9] Kawashima R, Koyama M, Ito H, et al. Normal cerebral perfusion of $99 \mathrm{mTc}-\mathrm{ECD}$ brain SPECT--evaluation by an anatomical standardization technique. Kaku Igaku 1996; 33: 69-72.

[10] Mena FJ, Mena I, Alamos BA, et al. SPECT Tc99m-HMPAO brain uptake in normal children: a comparison to normal elderly subjects. Alasbimn J 1998; 1: AJ01-3.

[11] George MS. Neuroactivation and Neuroimaging with SPET. New York: Springer-Verlag 1991.

[12] Eclipse Systems, Inc., nuclear psychiatry systems. available from: http://www.eclipsesys.com/PsychiatrySystems/tabid/247/default.as px

[13] Health Physics Society. Available from: http://www.hps.org/hpspublications/articles/dosesfrommedicalradiation.html.

[14] Kucuk NO, Kilic EO, Ibis E, et al. Brain SPECT findings in longterm inhalant abuse. Nucl Med Commun 2000; 21: 769-73.

[15] Jean-Bay E. The biobehavioral correlates of post-traumatic brain injury depression. J Neurosci Nurs 2000; 32: 169-76.

[16] Jacobs A, Put E, Ingels M, Bossuyt A. Prospective evaluation of technetium-99m-HMPAO SPECT in mild and moderate traumatic brain injury. J Nucl Med 1994; 35: 942-7.

[17] Baulieu F, Fournier P, Baulieu JL, et al. Technetium-99m ECD single photon emission computed tomography in brain trauma: comparison of early scintigraphic findings with long-term neuropsychological outcome. J Neuroimaging 2001; 11: 112-20.

[18] Kant R, Smith-Seemiller L, Isaac G, Duffy J. Tc-HMPAO SPECT in persistent post-concussion syndrome after mild head injury: comparison with MRI/CT. Brain Inj 1997; 11: 115-24.

[19] Goshen E, Zwas ST, Shahar E, Tadmor R. The role of 99TcmHMPAO brain SPET in paediatric traumatic brain injury. Nucl Med Commun 1996; 17: 418-22.

[20] Newberg AB, Alavi A, Payer F. Single photon emission computed tomography in Alzheimer's disease and related disorders. Neuroimaging Clin N Am 1995; 5: 103-23.

[21] Pimlott SL, Ebmeier KP. SPECT imaging in dementia. Br J Radiol 2007; 80 (Spec No 2): S153-9.

[22] Alexander GE, Prohovnik I, Sackeim HA, Stern Y, Mayeux R. Cortical perfusion and gray matter weight in frontal lobe dementia. J Neuropsychiatr Clin Neurosci 1995; 7: 188-96.

[23] Goto $\mathrm{H}$, Ishii $\mathrm{K}$, Uemura $\mathrm{T}$, et al. Differential diagnosis of dementia with Lewy Bodies and Alzheimer Disease using combined MR imaging and brain perfusion single-photon emission tomography. AJNR Am J Neuroradiol 2010; 31: 720-5.

[24] Bonte FJ, Weiner MF, Bigio EH, White CL, 3rd. Brain blood flow in the dementias: SPECT with histopathologic correlation in 54 patients. Radiology 1997; 202: 793-7.

[25] Alptekin K, Degirmenci B, Kivircik B, et al. Tc-99m HMPAO brain perfusion SPECT in drug-free obsessive-compulsive patients without depression. Psychiatr Res 2001; 107: 51-6.

[26] Lacerda AL, Dalgalarrondo P, Caetano D, Camargo EE, Etchebehere EC, Soares JC. Elevated thalamic and prefrontal regional cerebral blood flow in obsessive-compulsive disorder: a SPECT study. Psychiatr Res 2003; 123: 125-34.
[27] Hollander E. Obsessive-compulsive disorder-related disorders: the role of selective serotonergic reuptake inhibitors. Int Clin Psychopharmacol 1996; 11 (Suppl 5): 75-87.

[28] Carey PD, Warwick J, Niehaus DJ, et al. Single photon emission computed tomography (SPECT) of anxiety disorders before and after treatment with citalopram. BMC Psychiatry 2004; 4: 30.

[29] Amen DG, Carmichael B. Oppositional children similar to OCD on SPECT: implications for treatment. J Neurother 1997; 2: 1-6.

[30] Brockmann H, Zobel A, Joe A, et al. The value of HMPAO SPECT in predicting treatment response to citalopram in patients with major depression. Psychiatr Res 2009; 173: 107-12.

[31] Hoehn-Saric R, Schlaepfer TE, Greenberg BD, McLeod DR, Pearlson GD, Wong SH. Cerebral blood flow in obsessivecompulsive patients with major depression: effect of treatment with sertraline or desipramine on treatment responders and nonresponders. Psychiatr Res 2001; 108: 89-100.

[32] Mayberg HS, Brannan SK, Mahurin RK, et al. Cingulate function in depression: a potential predictor of treatment response. Neuroreport 1997; 8: 1057-61.

[33] Seminowicz DA, Mayberg HS, McIntosh AR, et al. Limbic-frontal circuitry in major depression: a path modeling metanalysis. Neuroimage 2004; 22: 409-18.

[34] Mayberg HS, Brannan SK, Tekell JL, et al. Regional metabolic effects of fluoxetine in major depression: serial changes and relationship to clinical response. Biol Psychiatry 2000; 48: 830-43.

[35] Saxena S, Brody AL, Maidment KM, et al. Cerebral glucose metabolism in obsessive-compulsive hoarding. Am J Psychiatry 2004; 161: 1038-48

[36] Diler RS, Kibar M, Avci A. Pharmacotherapy and regional cerebral blood flow in children with obsessive compulsive disorder. Yonsei Med J 2004; 45: 90-9.

[37] Wu JC, Gillin JC, Buchsbaum MS, et al. Sleep deprivation PET correlations of Hamilton symptom improvement ratings with changes in relative glucose metabolism in patients with depression. J Affect Disord 2008; 107: 181-6.

[38] Wu J, Buchsbaum MS, Gillin JC, et al. Prediction of antidepressant effects of sleep deprivation by metabolic rates in the ventral anterior cingulate and medial prefrontal cortex. Am J Psychiatry 1999; 156: 1149-58.

[39] Langguth B, Wiegand R, Kharraz A, et al. Pre-treatment anterior cingulate activity as a predictor of antidepressant response to repetitive transcranial magnetic stimulation (rTMS). Neuro Endocrinol Lett 2007; 28: 633-8.

[40] Dougherty DD, Weiss AP, Cosgrove GR, et al. Cerebral metabolic correlates as potential predictors of response to anterior cingulotomy for treatment of major depression. J Neurosurg 2003; 99: 1010-7.

[41] Oner P, Oner O, Aysev A, Kucuk O, Ibis E. Comparison of cerebral blood flow in children with obsessive compulsive disorder and attention deficit hyperactivity disorder. Turk Psikiyatri Derg 2008; 19: 13-8.

[42] Molina Rodriguez V, Montz Andree R, Perez Castejon MJ, et al. SPECT study of regional cerebral perfusion in neuroleptic-resistant schizophrenic patients who responded or did not respond to clozapine. Am J Psychiatry 1996; 153: 1343-6.

[43] Noel X, Sferrazza R, Van Der Linden M, et al. Contribution of frontal cerebral blood flow measured by $(99 \mathrm{~m}) \mathrm{Tc}$-Bicisate spect and executive function deficits to predicting treatment outcome in alcohol-dependent patients. Alcohol Alcohol 2002; 37: 347-54.

[44] Kanetaka H, Hanyu H, Hirao K, et al. Prediction of response to donepezil in Alzheimer's disease: combined MRI analysis of the substantia innominata and SPECT measurement of cerebral perfusion. Nucl Med Commun 2008; 29: 568-73.

[45] Mega MS, Dinov ID, Lee L, et al. Orbital and dorsolateral frontal perfusion defect associated with behavioral response to cholinesterase inhibitor therapy in Alzheimer's disease. J Neuropsychiatry Clin Neurosci 2000; 12: 209-18.

[46] Guedj E, Cammilleri S, Colavolpe C, et al. Predictive value of brain perfusion SPECT for ketamine response in hyperalgesic fibromyalgia. Eur J Nucl Med Mol Imaging 2007; 34: 1274-9.

[47] Amen DG, Hanks C, Prunella J. Predicting positive and negative treatment responses to stimulants with brain SPECT imaging. J Psychoactive Drugs 2008; 40: 131-8.

[48] Goethals I, Audenaert K, Jacobs F, et al. Brain perfusion SPECT in impulsivity-related personality disorders. Behav Brain Res 2005; 157: 187-92. 
[49] Amen DG, Hanks C, Prunella JR, Green A. An analysis of regional cerebral blood flow in impulsive murderers using single photon emission computed tomography. J Neuropsychiatr Clin Neurosci 2007; 19: 304-9.

[50] Amen DG, Prunella JR, Fallon JH, Amen B, Hanks C. A comparative analysis of completed suicide using high resolution brain SPECT imaging. J Neuropsychiatr Clin Neurosci 2009; 21: 430-9.

[51] Hales RE, Yudofsky SC. Textbook of neuropsychiatry and behavioral neurosciences, Fifth edition. Arlington, VA: American Psychiatric Publishing 2007.

[52] Devous MD, Sr., Leroy RF, Homan RW. Single photon emission computed tomography in epilepsy. Semin Nucl Med 1990; 20: 32541

[53] Titlic M, Basic S, Hajnsek S, Lusic I. Comorbidity psychiatric disorders in epilepsy: a review of literature. Bratisl Lek Listy 2009; 110: $105-9$.

[54] Joo EY, Hong SB, Tae WS, et al. Effect of lamotrigine on cerebral blood flow in patients with idiopathic generalised epilepsy. Eur J Nucl Med Mol Imaging 2006; 33: 724-9.

[55] Leiderman DB, Balish M, Bromfield EB, Theodore WH. Effect of valproate on human cerebral glucose metabolism. Epilepsia 1991; 32: 417-22.

[56] Blumer D. Neuropsychaitry of Personality disorders. Cambridge, MA: Blackwell Science 1995

[57] Gescher DM, Malevani J. Mood stabilizer in the psychopharmacotherapy of borderline personality disorder. Fortschr Neurol Psychiatr 2009; 77: 389-98

[58] Freo U, Pizzolato G, Dam M, Ori C, Battistin L. A short review of cognitive and functional neuroimaging studies of cholinergic drugs: implications for therapeutic potentials. J Neural Transm 2002; 109: 857-70.

[59] Insel T, Cuthbert B, Garvey M, et al. Research domain criteria (RDoC): toward a new classification framework for research on mental disorders. Am J Psychiatry 2010; 167: 748-51.

[60] Kuruoglu AC, Arikan Z, Vural G, Karatas M, Arac M, Isik E. Single photon emission computerised tomography in chronic alcoholism. Antisocial personality disorder may be associated with decreased frontal perfusion. Br J Psychiatry 1996; 169: 348-54.

[61] Raine A, Lencz T, Bihrle S, LaCasse L, Colletti P. Reduced prefrontal gray matter volume and reduced autonomic activity in antisocial personality disorder. Arch Gen Psychiatry 2000; 57: 119 27 ; discussion 28-9.

[62] Soderstrom H, Tullberg M, Wikkelso C, Ekholm S, Forsman A. Reduced regional cerebral blood flow in non-psychotic violent offenders. Psychiatry Res 2000; 98: 29-41.

[63] Volkow ND, Tancredi L. Neural substrates of violent behaviour. A preliminary study with positron emission tomography. $\mathrm{Br} \mathrm{J}$ Psychiatry 1987; 151: 668-73.

[64] Phillips ML. The emerging role of neuroimaging in psychiatry: characterizing treatment-relevant endophenotypes. Am J Psychiatry 2007; 164: 697-9.

[65] Little JT, Ketter TA, Kimbrell TA, et al. Bupropion and venlafaxine responders differ in pretreatment regional cerebral metabolism in unipolar depression. Biol Psychiatry 2005; 57: 2208.

[66] Beyenburg S, Mitchell AJ, Schmidt D, Elger CE, Reuber M. Anxiety in patients with epilepsy: systematic review and suggestions for clinical management. Epilepsy Behav 2005; 7: 16171 .

[67] Jones JE, Hermann BP, Barry JJ, Gilliam F, Kanner AM, Meador KJ. Clinical assessment of Axis I psychiatric morbidity in chronic epilepsy: a multicenter investigation. J Neuropsychiatr Clin Neurosci 2005; 17: 172-9.

[68] Reynolds CF, 3rd, Lewis DA, Detre T, Schatzberg AF, Kupfer DJ. The future of psychiatry as clinical neuroscience. Acad Med 2009; 84: 446-50.

[69] Downar J, Krizova A, Ghaffar O, Zaretsky A. Neuroimaging week: a novel, engaging, and effective curriculum for teaching neuroimaging to junior psychiatric residents. Acad Psychiatry 2010; 34: 119-24.

(C) Amen et al.; Licensee Bentham Open.

This is an open access article licensed under the terms of the Creative Commons Attribution Non-Commercial License (http://creativecommons.org/licenses/by-nc/3.0/) which permits unrestricted, non-commercial use, distribution and reproduction in any medium, provided the work is properly cited. 